\title{
Drugs and Human Information Processing
}

\author{
Enoch Callaway, M.D., Roy Halliday, Ph.D., Hilary Naylor, Ph.D., Lovelle Yano, B.A., \\ and Karen Herzig, B.A., J.D.
}

\begin{abstract}
Human performance on a choice-reaction time task (Eriksen task) has been simulated by a neural network. In simulations, the network captures many features of normal performance. In addition, changing gain in different layers produces changes that simulate different drug-induced changes. Data from a similar choicereaction time task have been reanalyzed to test some of the predictions derived from changing gain in different layers. Clonidine antagonizes norepinephrine and acetylcholine activities and changes speed-accuracy
\end{abstract}

tradeoff (i.e., increased frequency of errors at any specified reaction time). That is predicted when gain is reduced in lower layers (attention layer and input layer) of the network. By contrast, manipulating dopamine activity (with pimozide and amphetamine) changes reaction time without changing speed-accuracy tradeoff functions. That is predicted when gain is changed in the output layer of the network.

[Neuropsychopharmacology 10:9-19, 1994]
KEY WORDS: Neural networks; Human performance; Cognition; Psychopharmacology; Clonidine; Pimozide; Amphetamine

Neural networks offer ways to model drug effects on human information processing that seem superior to anything else currently available for that purpose. This paper is an introduction to the use of networks in psychopharmacology and has been written in the hope that others will help exploit the most promising new development on the "psycho" side of psychopharmacology in the past 50 years.

This enthusiastic preamble is justified on several grounds. First, the fundamental work to be described was done by Servan-Schreiber and Cohen and not by any of the present authors. Second, extra motivation is needed so that the reader will continue until some nontrivial results from network simulations can be demonstrated, and until it is apparent that no special mathematical skills are needed to use computer simulations of these networks.

The effort needed to understand neural networks

Address correspondence to: Enoch Callaway, M.D. San Francisco Veterans Affairs Medical Center (116T), 4150 Clement Street, San Francisco, California 94121.

Received May 28, 1993; revised August 24, 1993; accepted August 26, 1993. must be justified first by a demonstration of how these networks yield nontrivial results. To do that, a radically simplified network will be described and its practical value illustrated with data from human drug studies. This will show how the model provides a structure for organizing the data, how it suggests new ways of looking at the data, and how it aids in the formulation of new alternative hypotheses. After justifying the effort of learning about neural networks, a few more details of the particular network used here will be offered, along with some information on neural networks in general.

Human psychopharmacologists generally turn out data-driven studies. This is justified by the need to find treatments for diseases and to catalogue side effects. Although they may be content with atheoretical observations and with the limited models in general use, it is the lack of good models that has excluded the psycho side of psychopharmacology from the rapid development enjoyed by all other aspects of psychopharmacology. Indeed, since the psychopharmacologist's end product is a change in human information processing, one could compare pure neuroscientists to the drunk looking for his keys under the street light, rather than in the dark where he lost them. Neuroscientists in turn could argue that at least they find things, while those wholabor in the murky dark of human psychology have served as little more than a cheering section in the "psy- 
chopharmacological revolution" these past 50 or so years.

\section{THE ERIKSEN TASK AND MODEL}

Figure 1 illustrates a network used to simulate human performance on a choice-reaction time task described by Eriksen and Eriksen (1974). In the Eriksen task, subjects are asked to respond with a different hand to each of two target letters ( $\mathrm{S}$ and $\mathrm{H}$ ) that appear in the middle of a three- or five-letter stimulus array. In the compatible condition, all letters are identical (i.e., $\mathrm{HHHHH}$ or SSSSS), in the incompatible condition the central letter is different from the surrounding letters (i.e., $\mathrm{HHSHH}$ or SSHSS). All stimuli have an equal probability of being presented.

Coles and his group (Coles et al. 1985; Gratton et al. 1988) have used the Eriksen task to provide sophisticated studies of the relations between speed of processing and accuracy of response and have performed detailed studies of performance using supplemental psychophysiologic measures such as response force, electromyograms (EMGs), and event-related potentials (ERPs), (including P300 and motor readiness potentials). This group's work was based on the continuous flow theory (McClelland 1979; Eriksen and Schultz 1979). More recent work (Servan-Schreiber, 1990; Cohen et al. 1992) with parallel distributed processing (PDP) networks has captured features of Eriksen task performance that other theories have not been able to explain (Gratton 1993).

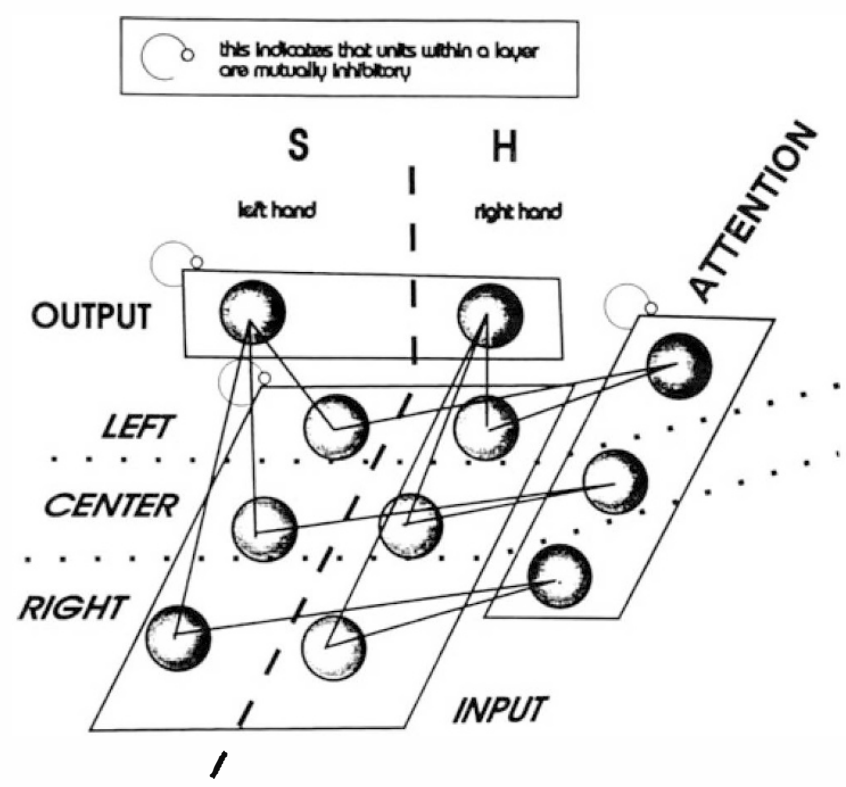

Figure 1. Diagram of simple eleven unit PDP network that simulates human performance on the Eriksen choice-reaction time task.
The network in Figure 1 has been simplified to use two instead of four flanking letters and contains the minimum number of units needed to represent the crucial task variables. Each of its 11 units sum its inputs and from that calculates an activation that supplies inputs to other units in the next cycle.

The units in the network are grouped into three layers (or modules): an attention layer, an input layer, and an output layer. The three units in the attention layer correspond to the three possible positions (left, center, or right) to which attention can be directed. Each attention unit is connected to the appropriate unit in the input layer. The six input layer units correspond to the six possible inputs ( $\mathrm{H}$ or $\mathrm{S}$ in three positions). All $S$ units in the input layer are connected to the S-response unit in the output layer, and all $\mathrm{H}$ units in the input layer are connected to the $\mathrm{H}$-response unit in the output layer. Connections between units in different layers are bidirectional and excitatory, so that units in different layers that are connected to each other mutually excite each other. All units within a layer inhibit each other. This sort of PDP network is referred to as an Inhibition and Activation Competition (IAC) network (McClelland and Rumelhart 1986; Rumelhart and McClelland 1986).

In simulations, continuous time is represented by discrete cycles. In the course of a cycle, the inputs to each unit are first computed from the activations of all appropriate units and then the activations of each unit are updated for the next cycle. The computation of activation from the summed inputs is simple but nonlinear and will be discussed later. Activation accumulates gradually and in parallel from input to output layers. Reaction time is taken as the number of cycles required for activation of a responselayer unit to reach some fixed threshold. Reaction time distributions and errors are produced by adding noise (i.e., inputting an independent random number with a Gaussian distribution to each unit on each cycle) and running trials over and over to accumulate the desired numbers of (simulated) reaction times and errors.

As the cycles are repeated, external activations applied to input and attention layers eventually increases activation in one or the other units in the output layer to the point at which threshold is reached and a response is recorded. To provide an initial elaboration of the model, the work of Cole's group has been used and relationships between ERP latencies and activations in portions of the network have been postulated. Thus, the activation of the input layer is assumed to be related to P300 latency. In a similar vein, the two units in the output layer can be thought of as representing the left and right motor systems, with the lateralized motor readiness potentials (LMRPs) from the contralateral sides reflect the activations of the two systems. Thus, the LMRP from the side contralateral to the respond- 
ing hand at the time of the response is a reflection of threshold. Prestimulus anticipatory buildup is reflected by the difference between the LMRPs at the time of stimulus presentation.

In the Gratton study (1988), instructions emphasize the importance of speeded responses and subjects are pushed to go faster until they make $15 \%$ to $30 \%$ errors. Subjects make more errors and are slower in the incompatible condition. Speed-accuracy functions are obtained when the probabilities of an accurate response (or probabilities of errors, each of which is 1.0 minus the probability of an accurate response) are plotted against reaction time. Note that the typical speedaccuracy curve rises sharply, then slowly approaches asymptote. Unfortunately, with the usual "be fast but accurate" instructions, most tasks are performed with reaction times well out on the asymptote so that a change in speed has very little effect on accuracy.

The shape of this speed-accuracy curve is not the same for the compatible and incompatible conditions (Fig. 2a). In the compatible condition, accuracy starts at $50 \%$ correct (random response) for very short reaction times and rises monotonically to an asymptote close to $100 \%$ correct. In the incompatible condition, in which performance is affected by inadvertent attention to the error-inducing flanking letters of the stimulus array, performance also starts at chance, but then drops below chance before it begins its rise to asymptote. This "dip" in the speed/accuracy curve reflects the domination of stimulus processing by the flanking letters at the very beginning of stimulus processing. It will be seen that the network model provides an explicit and quantitative way of representing that phenomenon.

Reaction times and errors from simulated human trials (cycles to threshold and errors) are divided into bins on the basis of reaction times (number of cycles). Frequency of errors is computed for each reaction-time bin. This procedure yields simulated reaction-time distributions and speed-accuracy curves for the compatible and incompatible conditions (Fig. 2b). Figure 2a shows the human data. It can be seen that the simulation captures many aspects of human performance: (1) the monotonic approach to asymptote of the accuracy curve in the compatible condition; (2) the dip in the accuracy curve of the incompatible condition; (3) the overall shape of the reaction-time distribution; and (4) the greater number of responses in the later bins in the incompatible condition than in the compatible condition.

\section{PSYCHOPHARMACOLOGICAL MODELING}

Most psychotropic drugs produce their effects by actions on neurotransmitters that can be considered context carriers rather than content carriers. Such transmitters are often released by neurons with small nuclei of origin that project widely to cortex and other structures. They tend to alter the target cell's response to other excitatory or inhibitory inputs (see Servan-Schreiber, 1990 for a review). Examples of context-setting transmitters include dopamine (DA), acetylcholine ( $\mathrm{ACh}$ ), serotonin (5-HT), and norepinephrine (NE). In PDP networks, the results of manipulating these context-setting transmitter systems is simulated by changing the gain parameter of the activation functions for various layers in a network (Servan-Schreiber et al. 1990; Cohen and Servan-Schreiber 1992). Increasing gain potentiates the unit's response, and reducing gain has the opposite effect. Decreasing gain in any layer produces results that are exactly symmetrical to the effects of increasing gain in that layer, and this predicts that antagonists should have opposite effects to those of agonists, to the extent that they affect the same receptors in the same brain region.
EMG Data from Gratton et al., (1988)
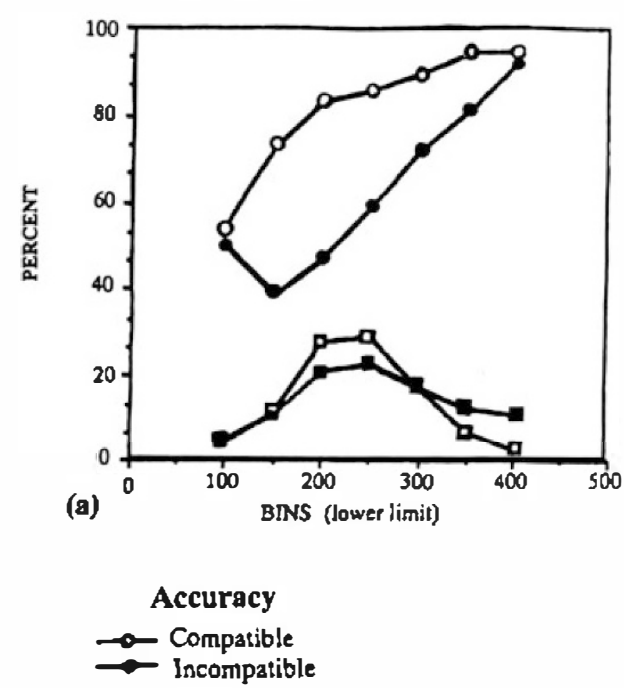

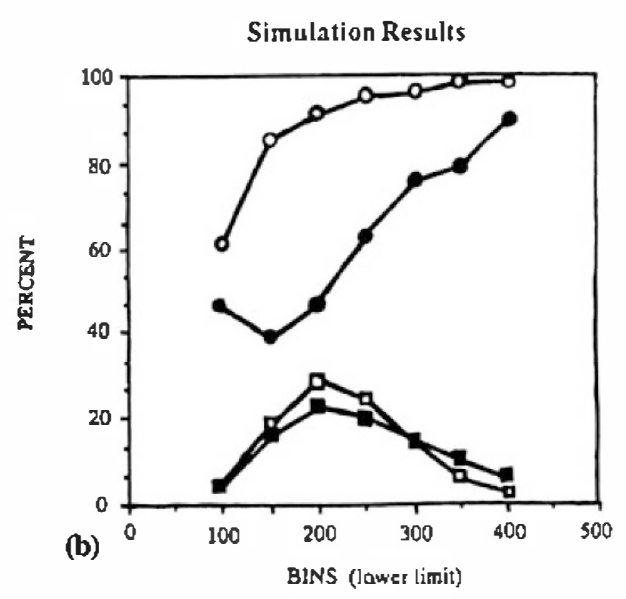

Reaction Time

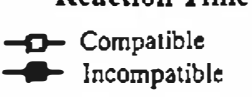

Figure 2. Speed and accuracy with compatible and incompatible versions of the Eriksen task. (a) Human Data (from Gratton et al. 1988). (b) Results of computer simulations with the network shown in Figure 1 (from Servan-Schreiber 1990). 
Increasing gain in the output layer increases amplitude of its output but does not change signal/noise ratios. As information about the stimulus accumulates over time, reaction time goes up and the probability of an error goes down (speed-accuracy tradeoff). At any time after the stimulus presentation, multiplying the output by a constant may cause the output to reach threshold and generate a response, but the error frequency (signal/noise ratio) will be the same function of time after stimulus (i.e., unchanged by the increased gain).

In contrast, when gain is increased by the same amount over the attention units only, the reaction-time distribution remains unchanged, whereas the speedaccuracy curve is shifted upwards. This pattern predicts no change in reaction time with an increase in accuracy (Fig. 3).

The complexities of drug actions at the cellular level and the multiple interacting neurotransmitter systems that mediate those actions are now so baroque (Nicoll et al. 1990) that neurobiology is no longer a good source of hypotheses about human information processing. What with so many known effects of any one transmit-
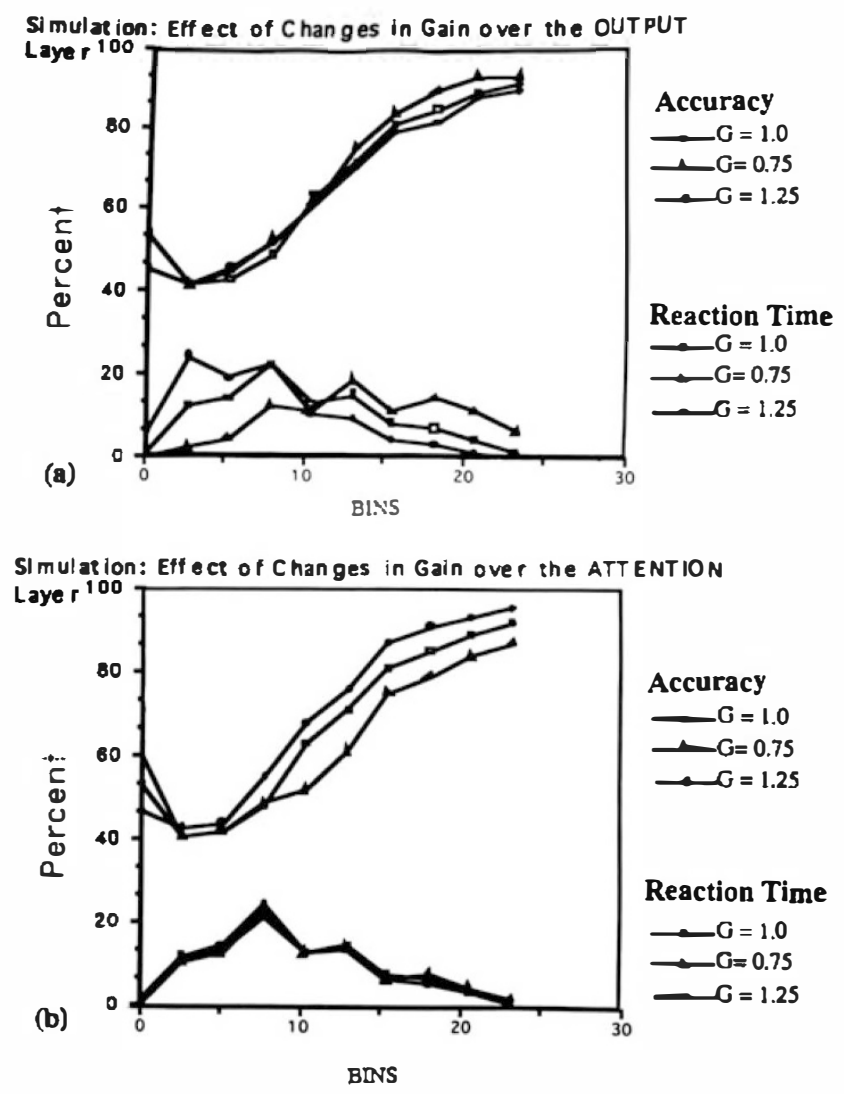

Figure 3. Simulations with changing gains. (a) Effects on speed and accuracy produced by changing gain in response (output) layer. (b) Effects on speed and accuracy produced by changing gain in attention layer (both from ServanSchreiber 1990). ter via its different receptors and with such a variety of reciprocal interactions at so many different levels, a clever neuroscientist should be able to make any drug effect on information processing sound plausible. At this stage, the crudest hypotheses concerning network gains and neurotransmitter systems are justified, as long as they generate testable hypotheses. Elaborate theoretical defenses of such preliminary hypotheses provide interesting academic exercises, but are not essential.

Nevertheless, there has developed a moderate consensus regarding certain transmitter-information processing relationships. Acetylcholine is thought to play a role in controlling attention (Brandeis et al. 1992; Callaway et al. 1992). Dopamine is thought to have effects on attention (via frontal and mesolimbic circuits) and on motor readiness (via nigrostriatal circuity; see Levin et al. 1990 for a discussion of the complexities of DA $D_{1}, D_{2}$, muscarinic- $A C h$, and nicotinic-ACh interactions in studies of radial-arm maze performance in rats). Norepinephrine has been thought to increase signal/ noise ratio, whereas 5-HT has been thought to increase threshold for motor activity (also see Clonninger 1987; Cohen and Servan-Schreiber 1992; Soubrie 1989; VanderWolff and Robinson 1989).

On one level, it seems unreasonable to expect any simple effects to arise from the complex interactions at the cellular level. However, it is possible that things may be simpler at the macro level where the simplified nets apply than at the micro level of the neurobiologist. How else could 11 units in the network capture any interesting features of a process in humans that must involve many millions of units?

\section{IMPORTANCE OF SPEED-ACCURACY ANALYSIS}

One of the first consequences of simulating choicereaction time tasks is that the necessity of speedaccuracy analysis becomes obvious. Reaction time can be changed by changing gains (i.e., changing the speed of stimulus and response processing). It can also be changed by making changes prior to stimulus presentation in threshold and levels of output activation in one or the other of the output units (presumably the simulacrum of anticipating a particular stimulus and readying the anticipated response). The disentangling of these alternatives by measuring EMGs, LMRPs, and other ERPs is illustrated in Gratton et al. (1988). Here, the crucial point is that without speed-accuracy analysis, there is no way of telling the difference between speeding processing throughout the network on the one hand and changing output variables that influencing the ultimate timing of the motor response on the other. 
In spite of powerful arguments for the crucial role of speed-accuracy tradeoff functions in understanding choice-reaction time studies (Wickelgren 1977), they have been ignored in psychopharmacology except for a few studies of alcohol (Jennings et al. 1976; Rundell and Williams 1979; Peeke et al. 1980; Glenn and Parsons 1991). There is a simple explanation for the relative absence of speed-accuracy studies in psychopharmacology. They are time consuming and difficult, because there must be a relatively large number of errors and some very fast reaction times to support any sort of statistical inference. The usual "Be fast but accurate!" instructions generally produce approximately $3 \%$ errors and almost no reaction times at the fast end of the speed-accuracy distribution. Cued-response techniques (Wood and Jennings 1976) have used response deadlines to elicit responses before processing is complete. They are not suitable for simple tasks because normal reaction times are too short for a cue to produce speeded (high-error) responses. Such excuses are no longer valid because Cole's group has shown that subjects can be taught to operate at approximately the $30 \%$ error level. Pilot studies showing that speeded-response training can be applied to drug studies and full-scale drug studies are underway using that technique.

\section{REANALYSIS OF SPEED-ACCURACY EFFECTS SEEN WITH THE STIMULUS EVALUATION-RESPONSE SELECTION [SE-RS] TASK}

There is a large body of data on how a number of drugs affect a choice-reaction time task (the SE-RS task) that shares some features with the Eriksen task. The SE-RS task is illustrated in Figure 4. Subjects were normal young (aged 16 to 31 years) adults who were first practiced on the task and then run on double-blind both pre and post drug and placebo, with test days at least 1 week apart. The SE-RS task differs from the Eriksen task in that (1) the responding finger is determined by target stimulus position (rather than by the nature of the target stimulus itself as in the Eriksen task) and (2) twolevels of response (Easy Resp, Hard Resp) and stimulus (Easy Stim, Hard Stim) complexities are used to provide four task conditions. Most importantly, accuracy was emphasized and feedback for errors was given so that error rates were generally low. The SERS task was developed for use with a serial information processing model. For further discussion of serial models, see van der Molen et al. (1991).

Those data have been reanalyzed using normalization to pool the responses of a group of subjects into one response speed distribution and one speed-accuracy curve. For each drug studied, reaction-time data from all subjects both pre and post placebo and drug
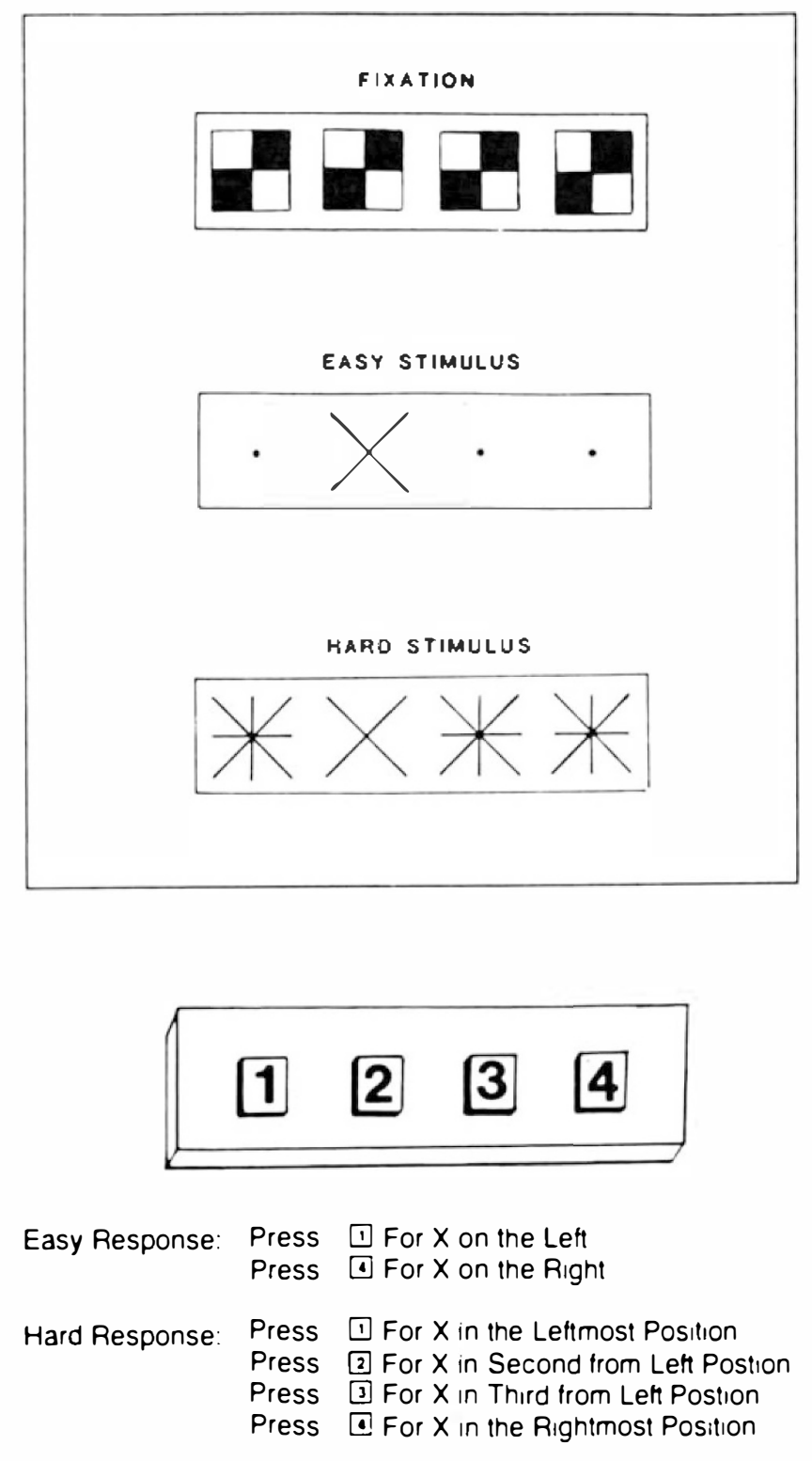

Figure 4. Stimulus Evaluation/Response Selection task based on serial model and used to obtain the data that are reanalyzed in this paper.

were normalized, so that for each of the four task conditions for each subject, the means were set to zero, and the standard deviations to 1.0. Note that for a given subject and task condition, the pre and post drug and placebo normalized reaction times would have different means and standard deviations. Pooling the normalized reaction times (Zs) allowed over 5000 response trials on each drug (drug/placebo/pre/post) condition to be analyzed together. In this way, data on the effects of clonidine, yohimbine, amphetamine, pimozide, nicotine, and cotinine have been reanalyzed. The reanalyses of studies with clonidine, pimozide, and amphetamine will be given here as illustrative examples. 
Table 1. Drug Doses and Subject Characteristics

\begin{tabular}{lcc}
\hline Drug & $\begin{array}{c}\text { Dose } \\
\text { (mg) }\end{array}$ & $\begin{array}{c}\text { Subject } \\
\text { Characteristic }\end{array}$ \\
\hline Clonidine & 0.2 & $21-30$ yrs old \\
Pimozide & 4.0 & $21-37$ yrs old; \\
15 yrs educ. & $21-30$ yrs old; \\
mamphetamine & 10.0 & malenly \\
\hline
\end{tabular}

All drugs were given by mouth and testing began 75 minutes after dosing. There were 12 subjects in each cohort. Numbers of trials varied because the program was set to collect a specific number of trials without eye movements for ERP analysis (Table 1).

Pooling normalized data may obscure important relationships, but it is unlikely to result in overestimating the significance of observations. Nevertheless, posthoc analysis of pooled data from an experimental paradigm that differs from the Eriksen task is clearly not ideal. However, these reanalyses are justified on two grounds. First, they are presented as pilot studies that will require replication. Second, the results are so striking and so suggestive of the power inherent in this approach that they may motivate others to begin exploring the use of neural network models on psychopharmacology.

The Gratton et al. (1988) data and the simulations (Fig. 2) display reaction time as traditional frequency distributions. There, points on the horizontal axis are equidistant (in terms of reaction time). Although conventional, this display has several shortcomings. First, the eye tends to underestimate differences between curves that are steeply rising and falling. Thus, there is an illusion in Figure 5 that the differences between the postplacebo and postclonidine curves are greatest at the mode. Second, magnitudes of differences can be misleading because there are fewer cases at the tails than nearer the modes of the distributions. Thus, bins near the tails will have larger standard deviations of the means.

Both problems are avoided by sorting normalized reaction times (and associated error frequencies) into equal sized bins. In Figure $6 \mathrm{a}$ and $6 \mathrm{~b}$ each bin contains 2000 trials from both pre and post placebo and clonidine. Only postdrug and postplacebo are shown for clarity. With the data displayed in this fashion, it is much more apparent that with clonidine, the change in reaction time is large and it is not restricted to any part of the distribution. Figure $6 \mathrm{~b}$ shows the effect of clonidine on the speed-accuracy function. Again, the bins are of equal size rather than of equal reaction-time range, and the increase in error frequency after clonidine is readily apparent.

Clonidine slows reaction times and increases error frequency throughout the distribution. This effect on

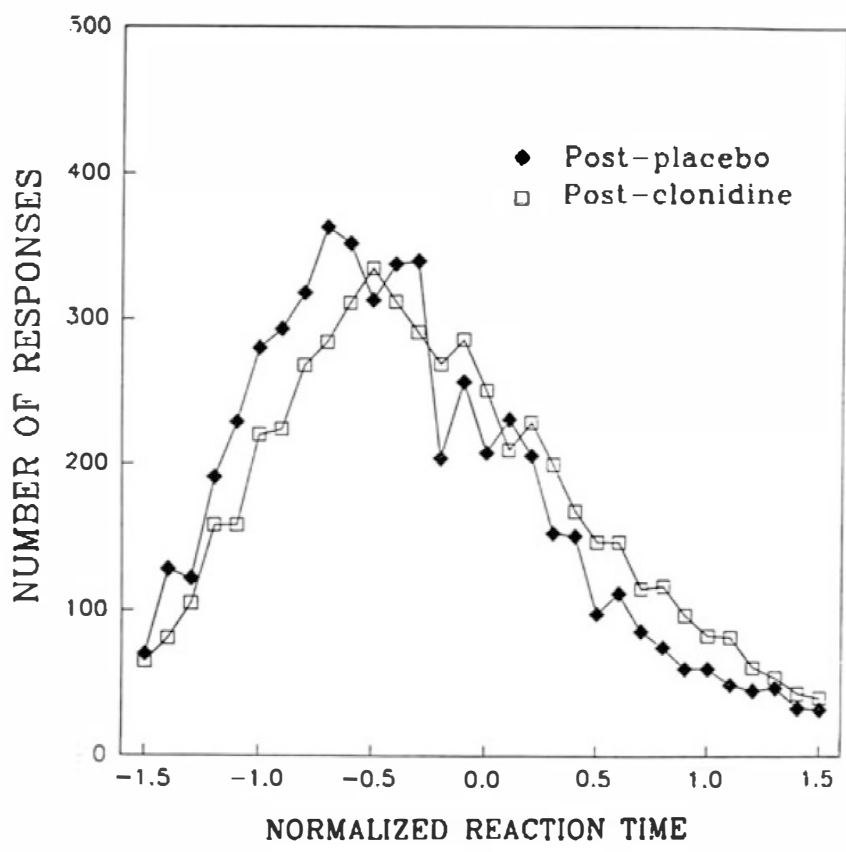

Figure 5. Distribution of response speeds postplacebo and postclonidine. Data are shown as normalized reaction times. Normalization was done using both pre and post placebo and drug, but only values for post are shown for clarity. Responses are arranged in bins spanning equal intervals on the normalized reaction time axis. Thus, the horizontal axis is similar to those in Figures 2 and 3. This is the conventional frequency distribution curve but with real data. As bins go from the mode toward the tails, they contain progressively fewer cases, and this results in progressively larger standard deviations of the means.

errors is predicted when there is a reduction of gain in the attention and/or input layers. Clonidine is an $\alpha$-noradrenergic agonist with a predominantly presynaptic action. It not only reduces NE output but also reduces ACh output, and it provides more protection against anticholinesterases than does scopolamine (Buccafusco and Aronstam 1987). On either score, it would be a good bet if one wished to reduce attention-layer gain.

In Figure $6 \mathrm{c}$ and $6 \mathrm{~d}$, it can be seen that pimozide also slows reaction time (fewer postdrug responses at the fast end of the distribution and more at the slow end). However, pimozide does not change speedaccuracy functions. No speed-accuracy change is expected when output layer alone is being affected, and as a DA antagonist, pimozide was expected to primarily affect the output layer.

Pimozide should have an effect that is nearly the opposite to that of d-amphetamine, and that is exactly what is shown in Figure 6e and 6f. Amphetamine speeds reaction time but, like pimozide, it has no effect on error frequency as a function of reaction time. 


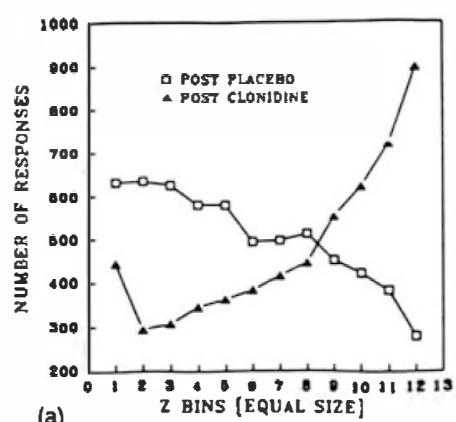

(a)
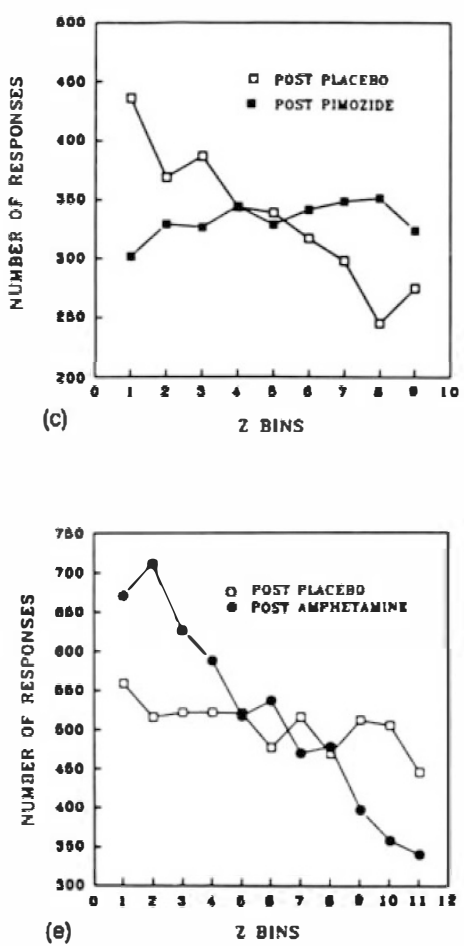
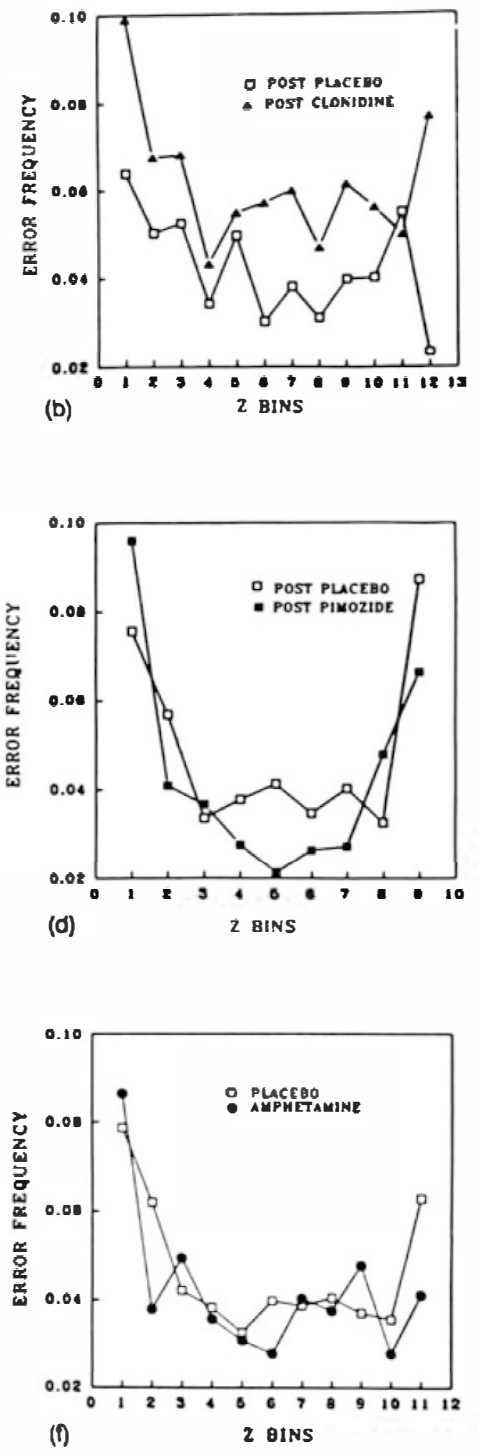

Figure 6. Comparison of drug effects on SE/RS task. (a) These are the same data as in Figure 5 but with bins containing equal numbers of responses (reaction times widely spaced at tails of the distribution). Note how the slowing postclonidine (fewer postclonidine responses at fast end of distribution) is much more apparent in this figure. (b) Accuracy postplacebo and postclonidine. Note reduction of accuracy by clonidine. (c) Distributions of responses (ordered according to normalized reaction times) postplacebo and postpimozide. The slowing postpimozide is similar to that produced by clonidine. (d) Accuracy postplacebo and postpimozide. In contrast to clonidine, note the absence of any effect on accuracy. (e) Normalized reaction times postplacebo and postamphetamine. Note speeding of reaction time. (f) Accuracy postplacebo and postamphetamine. Like pimozide, there is no effect on accuracy.

\section{IMPLICATIONS}

The ability of the network simulations to suggest ways to examine data, and to explain results is remarkable, both in the examples given here and in other more complex situations. Thus, the model predicted that a DA antagonist (pimozide) would slow reaction time without changing speed-accuracy relationships, and that an agonist (amphetamine) would have the opposite effect. That is exactly what has been observed. It predicted that clonidine, by reducing both $\mathrm{ACh}$ and $\mathrm{NE}$ activity, would increase errors at all reaction times and that too has been observed.

At yet another level, the model suggests some deeper alternative explanations for the effects of DA agents (pimozide and amphetamine) on reaction time. Thus, there may have been changes in output level gain after stimulus presentation (as in the simulations) or changes in the output layer may have affected prestimulus (anticipatory) motor readiness. Finally, the drugs may have had direct or indirect effects on threshold. These alternatives will be evaluated in the studies under way. Motor readiness can be evaluated using motor readiness potentials as indications of relative activations of output units. Changes in threshold will be reflected in motor readiness potential asymmetry at the time of response. The model can be used to generate numerical estimates of how changes in gain, threshold, and prestimulus buildup would interact to change reaction time. Evaluating these numerical predictions will be much more powerful than simply testing the null hypothesis.

There are many other aspects of the model that are intriguing. One of the most interesting properties (and one that is commonly encountered in working with networks) is the way that apparently complex phenomena 
arise quite simply in the operation of the network. The way the network generates the "paradoxical" below chance performance at the fast end of the speed-accuracy curve in the incompatible condition is one example. that will be dealt with at greater length in the next section.

With respect to the pharmacologic issues, the current version of the network suggests other drug studies. Would 5-HT agents alter threshold? If so, a 5-HT agonist would slow reaction time without changing the speedaccuracy function. The model will generate several alternative hypotheses as it did for the amphetamine data. This could be used to examine the differences between the effects of drugs acting on different 5 -HT receptors.

With respect to clonidine, although there are similarities between its effects and those of scopolamine, differences between the effects of the two drugs on other measures of human information processing have been reported (Callaway et al. 1991). How would the effects of the two drugs differ on the Eriksen task with speed instructions? The effects of clonidine should be imitated more by increasing noise input (by virtue of its effect on NE), whereas both clonidine and scopolamine should produce effects simulated by reducinggain in the attention layer (through their actions on ACh).

At this point, a flaw in the current network will be pointed out to show how the network provides a framework for future elaboration. Clearly, the radically simplified network described here cannot simulate all the complexities of the human central nervous system. The fact that it captures so many features suggests that its basic structure can indeed sustain further elaboration. The testing and elaboration of this (and other) networks for predicting drug effects offers a fascinating challenge, and the offering of this challenge to other colleagues is the principal reason for this essay.

One of the model's failures will already be apparent to the careful reader who compared Figure 3 (simulation of changed gain in attention layer) and Figure 5 (the observed effect of clonidine). The simulation predicted major changes in error rate-reaction time functions with minimal changes in reaction time. The drug data showed the predicted change in speedaccuracy function, and thus provided a useful contrast to the effects of DA manipulations. However, the large change in reaction time suggests some additional effect on output operations. Does this mean a change in output layer gain via some nonobvious neuropharmacologic interaction? Perhaps, and if so, the slowing should be apparent in every reaction time regardless of the nature of its preceding reaction time. Alternatively, some work by Cole's group suggests that, when people make errors, they slow down their next few reaction times. This is particularly the case when "fast but accurate" instructions are given (as was the case with the reanalyzed data). Note here that there are a number of alter- native hypotheses that can be explicitly modeled and tested. Perhaps clonidine has some unexpected effects on striatal functioning, although its anticholinergic effects would be expected, if anything, to imitate a DA agonist. Perhaps the model will need an additional mechanism that will respond to errors by changing one or more parameters if it is to simulate an error-avoiding response. Perhaps "speed" instructions will eliminate the error-correcting effect?

\section{MORE ABOUT NEURAL NETWORKS}

This essay is not the place for a discussion of the relationships between these radically simplified networks and either the underlying brain circuits or the underlying human information processing operations. The issues involved are both too important and too complex for this introduction. A good overview of some of the more important issues can be found in Smolensky (1988) and Clark (1989).

As for the topic of neural networks themselves, the literature is enormous, because they now have great economic significance in the computer industry. There are a number of societies and several monthly journals devoted to the topic. For the beginner, the McClelland and Rumelhart (1988) workbook and disks are probably the best way to get started. However, some of the issues glossed over in the preceding sections can be usefully discussed here, as they will give some indications as to the sorts of surprises that await those who are stimulated to investigate the topic in more depth.

First, a discussion of the way a unit processes its input was postponed until some interest in it could be generated. Obviously, the units are not simply summing devices. Like real neurons, they have maximum and minimum activations. The closer to maximum, the less effect excitation has, and the closer to minimum, the less effect inhibition has. To achieve this effect in a network takes only some very simple arithmetic. For example, the excitatory input is multiplied by the difference between existing excitation and maximum excitation. Then, there is a decay function, so that the unit drifts back toward resting when there is no input. This occurs faster when excitation is high and slows as excitation decreases. This is accomplished by multiplying current excitation by a decay constant that is less than 1. All this nonlinearity bestows some nonobvious properties on the networks. For example, changing gain in one layer does not change the signal/noise ratio in its output, whereas changing gain in the first of two layers does change signal/noise ratio in the output of the second layer. That explains why changing output gain fails to alter speed-accuracy tradeoff functions.

A particularly useful property of networks is that they can be examined in detail at each step that occurs 


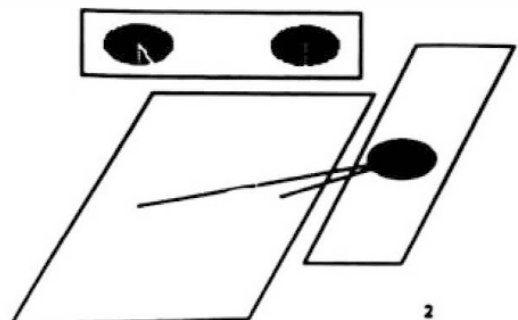

2

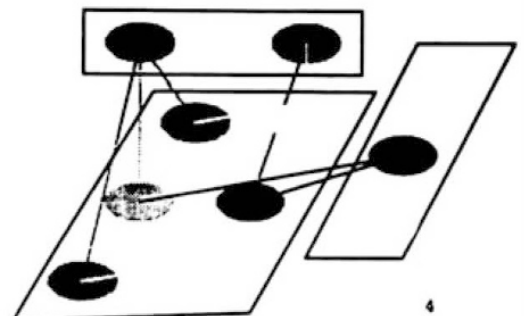

4
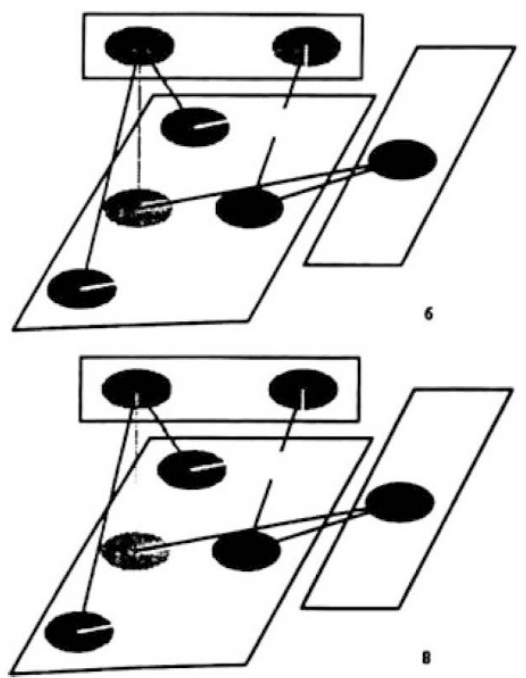
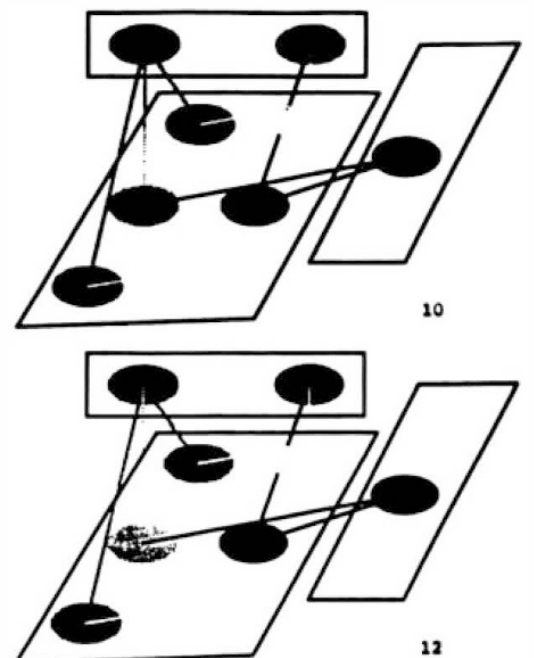

12

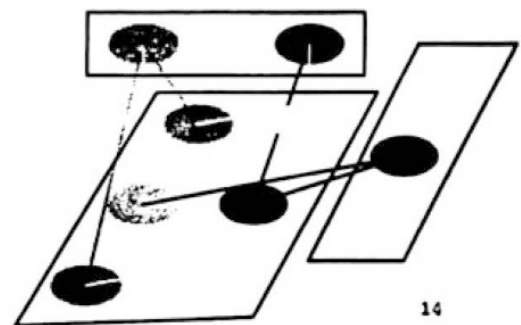

14

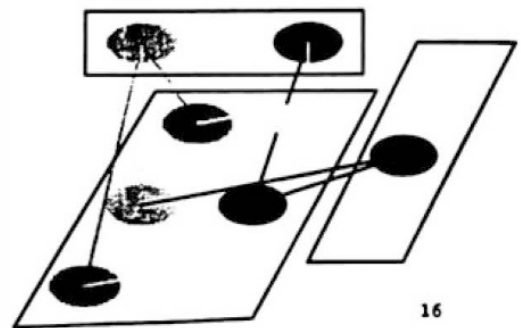

Figure 7. Sequence of activations in network under "incompatible" conditions. The units are as shown in Figure 1. As units become activated, they are shaded more darkly. Note the initial buildup of the incorrect response. in simulating an information processing operation. Figure 7 shows graphically how the incompatible condition generates below-chance accuracy when reaction time is pushed close to its minimum. The figures are drawn from a no-noise incompatible-stimulus simulation run, and cycle numbers are shown in the lower right corner of each frame. Shadings of the units indicate their activations. Before the stimulus is presented, anticipatory attention to the center position is simulated by activation of the attend-center unit. Then an incompatible array is presented and the initial excitation of the "wrong" response can be seen. This results in an above-chance probability of a wrong response when the response is emitted before the activation of the "correct" response takes over.

There is obvious face validity in the use of a random number generator to simulate noise and in the use of repeated runs on the computer to simulate the repeated trials of a human study. However, Pfaff et al. (1990) have pointed out that the activations of output units can be used to compute response probabilities. The noise distribution determines the response distribution in a simulation. If noise is largely in the output, then the noise distribution can be used to assign probabilities of responses at various reaction times, and the activations of the units (indicated by shading of output units in Fig. 7) can be used to calculate the probabilities of correct and erroneous responses at any given cycle. For a more general treatment, including the effects of noise at other levels, see McClelland (1993).

So far, the fact that even the simplest network has a larger number of parameters has been glossed over. Among these parameters are the gains in each level, connections and their weights between units both within and between layers, and values for inhibition, excitation, external input, and decay. There are various methods for setting these parameters. More sophisticated networks can be made to "leam" their parameters. There are several methods for this that are in use, and many of the networks used in commercial applications 
do just that. The other approach is to use trial and error to produce a network that performs as desired. It would seem that with so many parameters, almost any imaginable sort of performance could be simulated. In fact, the basic architecture of the network puts many limits on possible operations. In addition, to avoid the suspicion that results are post hoc and, hence, meaningless, parameters can be first set to match normal performance, and then a search can be made for single parameters that can be changed to match different drug effects. In the network described here, the trial and error work to match normal performance was done "by hand." More recently, polynomial (polytope) programs have been used to do the trial and error work automatically (Everitt 1987; Murray 1989).

\section{CONCLUSION}

The lack of good models has excluded the psycho side of psychopharmacology from the rapid development enjoyed by all other aspects of psychopharmacology. Neural network models offer a better chance of rescuing the study of human psychologic responses to drugs than anything else currently available.

To illustrate this, human performance on a choicereaction time task (Eriksen task) has been simulated by the neural network described here. Not only do the network simulations capture many features of normal performance but changing gains in different layers produce changes that are expected to simulate certain druginduced changes. Data from a similar choice-reaction time task have been reanalyzed to test some of the predictions derived from changing gains in different layers. Manipulating DA activity (with pimozide and amphetamine) changes reaction time without changing speed-accuracy tradeoff functions. This is predicted from simulations with changed output operations. Output operations include gain in the output layer, threshold for response, and anticipatory activation of the output layer. By way of contrast, an NE and ACh antagonistchanges speed-accuracy tradeoff, as predicted from changing gain in lower layers (attention layer and input layer).

Implications of the above observations are discussed. It is shown how they can be used for organizing psychopharmacologic observations, for formulating quantitative predictions from alternative hypotheses, and for designing new experiments.

\section{ACKNOWLEDGMENT}

Versions of this paper have been given at the 1992 meeting of the West Coast College of Biological Psychiatry, the 1993 meeting of the Psychiatric Research Society, and portions of it will appear in Peslow E, Tomoro N (eds), The Effects of Medication and Psychopathology on Memory. Washington DC, American Psychiatry Association Press. Special thanks are due to Dr. Servan-Schreiber and Ms. Morgan BenowitzFredricks.

\section{REFERENCES}

Brandeis D, Naylor H, Halliday R, Callaway E, Yano L (1992): Scopolamine effects on visual information processing, attention and ERP map latencies. Psychophysiology 29:315-336

Buccafusco JJ, Aronstam RS (1987): Adrenergic agonists protect against soman, an irreversible acetylcholinesterase inhibitor. Toxicol Lett 38:167-176

Callaway E, Halliday R, Naylor H (1991): Clonidine and scopolamine: Differences and similarities in how they change human information processing. Prog Neuropsychopharmacol Biol Psychiatry 15:497-502

Callaway E, Halliday R, Naylor H (1992): Cholinergic activity and restricted information processing. Biol Psychol 33:1-22

Clonninger CR (1987): A systematic method for clinical descriptions and classifications of personality variants. A proposal. Arch Gen Psychiatry 44:573-588

Cohen JD, Servan-Schreiber D, McClelland JL (1992): A PDP approach to automaticity: Principles and models. Am J Psychol 105:239-269

Coles MGH, Gratton G, Bashore TR, Eriksen CW, Donchin $E$ (1985): A psychophysiological investigation of the continuous flow model of human information processing. J Exp Psychol 11:529-553

Clark, A (1989): Microcognition. Cambridge MA, MIT Press

Eriksen BA, Eriksen CW (1974): Effects of noise letters upon the identification of target letter in a non-search task. Percept Psychophys 16:143-149

Eriksen CW, Schultz DW (1979): Information processing in visual search; A continuous flow conception and experimental results. Percept Psychophys 25:249-263

Everitt B (1987): Introduction to Optimization Methods and their Applications in Statistics. London, Chapman and Hall

Glenn SW, Parsons OA (1991): The effects of alcohol and instructional conditions on speed/accuracy tradeoffs. Alcoholism: Clin Exp Res 15:612-619

Gratton G, Coles MGH, Sirevaag EJ, Eriksen CW, Donchin E (1988): Pre- and post-stimulus activation of response channels: A psychophysiological analysis. J Exp Psychol 14:331-344

Halliday R, Callaway E, Naylor H, Gratzinger P, Prael R (1986a): The effects of stimulant drugs on information processing in elderly adults. J Gerontol 41:748-757

Halliday R, Naylor H, Callaway E, Yano L, Walton P (1986b): $\mathrm{D}$-amphetamine speeds both stimulus and response processing. Eighth Intemational Conference on Event-Related Potential of the Brain. Stanford, CA

Halliday R, Naylor H, Callaway E, Yano L, Walton P (1987): What's done can't always be undone: The effects of stimulant drugs and dopamine blockers on information pro- 
cessing. Current Trends in Event Related Potential Research (EEG Suppl 40), New York, Elsevier

Halliday R, Callaway E, Lannon R (1989a): The effects of clonidine and yohimbine on human information processing. Psychopharmacology 99:563-566

Halliday R, Callaway E, Naylor H, Brandeis D, Yano L (1989b): The comparative effects of $d$-amphetamine and noradrenergic drugs on human information processing. Presented at the 29th Annual Meeting Society for Psychophysiological Research, New Orleans, Louisiana

Jennings RJ, Wood CC, Law rence BE (1976): Effects of graded doses of alcohol on speed-accuracy trade off in choice reaction time. Percep Psychophys 19:85-91

Levin ED, McGurk SR, Rose JE, Butcher LL (1990): Cholinergic-dopaminergic interactions in cognitive performance. Behav Neural Biol 54:271-299

Libet B, Mochida S (1988): Long-term enhancement (LTE) of post-synaptic potentials following neural conditioning in mammalian sympathetic ganglia. Brain Res 473:271282

McCarthy G, Donchin E (1981): A metric for thought: A comparison of P3 latency and reaction time. Science 211:77-80

McClelland JL (1979): On the time relations of mental processes: An examination of processes in cascade. Psychol $\operatorname{Rev} 86: 287-330$

McClelland JL (1993): Toward a theory of information processing in graded, random, interactive networks. In Meyer DR, Komblum S (eds), Attention and Performance XIV. Cambridge, MA, MIT Press

McClelland JL, Rumelhart DE (1986): Parallel Distributed Processing Explorations in the Microstructure of Cognition, Volume II. New Jersey, Erlbaum Publishers

McClelland JL, Rumelhart DE (1988): Explorations in Parallel Distributed Processing. Cambridge, MA, MIT Press

Murray W (1989): Practical Optimization. San Diego, Academic Press

Naylor H, Halliday R, Callaway E (1985): The effects of methylphenidate on information processing. Psychopharmacology 86:90-95

Naylor H, Callaway E, Halliday R (1993): Biological correlates of human information processing. In Vernon AP (ed), Biological Approaches to the Study of Human Intelligence. New Jersey, Ablex Publishing Corp

Nicoll RA, Malenka RC, Kauer JA (1990): Functional comparison of neurotransmitter receptor subtypes in mammalian central nervous system. Physiol Rev 70:513-565

Peeke S, Callaway E, Jones RT, Stone GC, Doyle J (1980): Combined effects of alcohol and sleep deprivation in normal adults. Psychopharmacology 67:279-287

Pfaff RH, Van der Heuden AHC, Hudson PTW (1990): SLAM:
A connectionistic model for attention in visual selection tasks. Cogn Psychol 22:273-341

Rumelhart DE, McClelland JL (1986): Parallel Distributed Processing Explorations in the Microstructure of Cognition, Volume I. New Jersey, Erlbaum Publishers

Rundell CH, Williams HL (1979): Alcohol and speed-accuracy tradeoff. Hum Fact 21:433-443

Servan-Schreiber D (1990): From physiology to behavior: Computational models of catecholamine modulation of information processing (Ph.D. Thesis). Technical Report CMU-CS-90-167, School of Computer Science, Carnegie Mellon University

Servan-Schreiber D, Printz H, Cohen JD (1990): A network model of catecholamine effects: Gain signal-to-noise ratio and behavior. Science 249:892-895

Smolensky P (1988): On the proper treatment of connectionism. Behav Brain Sci 11:1-74

Sternberg S (1969): The discovery of processing stages: Extensions of Donder's method. In Koster WC (ed), Acta Psychologica (Vol 30) Attention and Performance II. Amsterdam, Elsevier, pp 276-315

Soubrie P (1989): Reconciling the role of central sertonin neurones in human and animal behavior. Brain Behav Sci 9:319-364

Takakuwa KM, Callaway E (1991): Scopolamine and physostigmine do not alter visual detection of change: Relationship to a model of lateral geniculate operation. Neuropsychobiology 24:185-191

Tecce JJ, Savignano J, Cole JO (1978): Drug effects on contingent negative variation and eye blinks: The distractionarousal hypothesis. In Lipton MN, DiMascio A, Killam KF (eds), Psychopharmacology: A Generation of Progress. New York, Raven Press, pp 745-758

van der Molen MW, Bashore TR, Halliday R, Callaway E (1991): Chronopsychophysiology: Mental chronometry augmented by physiological time markers. In Jennings JR, Coles MGH (eds), Handbook of Cognitive Psychophysiology. Chichester, Sussex, John Wiley \& Sons

Vanderwolf CH, Robinson TE (1981): Reticulo-cortical activity and behavior: A critique of the arousal theory and a new synthesis. Behav Brain Sci 4:459-475

Wickelgren W (1977): Speed-accuracy tradeoff and information processing dynamics. Acta Psychologia 41:67-87

Wood CC, Jennings JR (1976): Speed-accuracy tradeoff functions in choice reaction times: Experimental designs and computational procedures. Percept Psychophys 19:92102

Yano LM, Takakuwa KM, Perkins D (1990): Stimulus duration affects P3 latency and reaction time. Psychophysiology (Suppl) 27 (4A); S75 